\title{
Dielectric Metamaterials with Toroidal Dipolar Response
}

\author{
Alexey A. Basharin, ${ }^{1,2,{ }^{*}}$ Maria Kafesaki, ${ }^{1,3}$ Eleftherios N. Economou, ${ }^{1,4}$ Costas M. Soukoulis, ${ }^{1,5}$ \\ Vassili A. Fedotov, ${ }^{6}$ Vassili Savinov, ${ }^{6}$ and Nikolay I. Zheludev ${ }^{6,7}$ \\ ${ }^{1}$ Institute of Electronic Structure and Laser (IESL), Foundation for Research and Technology \\ Hellas (FORTH), P.O. Box 1385, 71110 Heraklion, Crete, Greece \\ ${ }^{2}$ National Research University "Moscow Power Engineering Institute," 112250 Moscow, Russia \\ ${ }^{3}$ Department of Materials Science and Technology, University of Crete, 71003 Heraklion, Greece \\ ${ }^{4}$ Department of Physics, University of Crete, 71003 Heraklion, Greece \\ ${ }^{5}$ Ames Laboratory-USDOE and Department of Physics and Astronomy, Iowa State University, \\ Ames, Iowa 50011, USA \\ ${ }^{6}$ Optoelectronics Research Centre and Centre for Photonic Metamaterials, \\ University of Southampton, Highfield, Southampton SO17 1BJ, United Kingdom \\ ${ }^{7}$ Centre for Disruptive Photonic Technologies, Nanyang Technological University, \\ Singapore 637371, Singapore \\ (Received 7 November 2013; revised manuscript received 16 November 2014; published 27 March 2015)
}

\begin{abstract}
Toroidal multipoles are the terms missing in the standard multipole expansion; they are usually overlooked due to their relatively weak coupling to the electromagnetic fields. Here, we propose and theoretically study all-dielectric metamaterials of a special class that represent a simple electromagnetic system supporting toroidal dipolar excitations in the $\mathrm{THz}$ part of the spectrum. We show that resonant transmission and reflection of such metamaterials is dominated by toroidal dipole scattering, the neglect of which would result in a misunderstanding interpretation of the metamaterials' macroscopic response. Because of the unique field configuration of the toroidal mode, the proposed metamaterials could serve as a platform for sensing or enhancement of light absorption and optical nonlinearities.
\end{abstract}

DOI: 10.1103/PhysRevX.5.011036

\section{INTRODUCTION}

The toroidal dipole is a peculiar elementary current excitation corresponding to electrical currents circulating on a surface of a gedanken torus along its meridians (so-called poloidal currents). The toroidal dipole was first considered in 1958 by Zel'dovich, who suggested that such excitation produced by static currents (anapole), if supported by atomic nuclei, could explain parity violation in the weak interaction [1]. Since then, the existence of the static toroidal dipole has been predicted and its importance has been discussed for a number of solid-state systems including ferroelectric and ferromagnetic nano- and microstructures, multiferroics, macromolecules, molecular magnets, etc. [2-9]. The dynamic toroidal dipole is less known. Although it was shown to radiate electromagnetic fields, just as the conventional dynamic multipoles do, the toroidal dipole is not included in the standard multipole expansion of charge-current excitations and is thus often omitted from considerations in classical electrodynamics [10,11].

*basharin@iesl.forth.gr

Published by the American Physical Society under the terms of the Creative Commons Attribution 3.0 License. Further distribution of this work must maintain attribution to the author(s) and the published article's title, journal citation, and DOI.
Subject Areas: Metamaterials, Photonics, Plasmonics

Being physically distinct from the dynamic electric dipole (which is produced by oscillating charge density rather than currents), the toroidal dipole emits radiation with the same angular momentum and parity properties, which is therefore indistinguishable from that of an electric dipole for any distant observer [12]. There are, however, fundamental differences between the two types of radiation: The radiated power for electric and toroidal dipoles scales as, respectively, $\omega^{4}$ and $\omega^{6}[10,11,13]$; also, the vector-potential fields corresponding to the electric and toroidal dipolar emission do not coincide and the difference cannot be removed by gauge fixing [12].

Other intriguing phenomena expected in the presence of toroidal dipolar excitations include the violation of the action-reaction equality [14], nonreciprocal refraction of light [15], and the generation of a propagating nontrivial vector potential in the complete absence of electromagnetic fields [12]. The existence of the dynamic toroidal dipole also indicates that care should be taken while establishing a relation between the far-field properties of an electromagnetic source and the charge-current distribution forming the source. It applies to many domains of science dealing with electromagnetic interactions and, in particular, for nanophotonics and plasmonics where the topology of chargecurrent excitations controls the enhancement of local optical fields [16-24]. Furthermore, given the explicit 
toroidal topology of a great number of biologically important macromolecules and protein complexes [25-27], it is not unreasonable to expect that electromagnetic interactions involving the toroidal dipole (and higher toroidal multipoles) could play a special role in nature.

The detection of toroidal excitations is challenging [28]. The dynamic toroidal dipole interacts with curl $\mathbf{B}$ and is weakly coupled to free space, while its manifestation can be naturally masked by much stronger electromagnetic effects due to electric and magnetic dipoles and even an electric quadrupole. Experimental observation of the toroidal response has become possible only recently with use of the metamaterial concept [29]. This concept enables us to access to novel and exotic optical phenomena (such as negative refraction and cloaking [30-36]) by controlling the symmetry and character of electromagnetic response through artificially structuring media on a subwavelength scale. The toroidal dipolar response was demonstrated in a metamaterial composed of specially designed complex metallic electromagnetic scatterers (metamolecules) of toroidal topology, where electric and magnetic dipolar excitations had been suppressed, while toroidal response was spectrally isolated and resonantly enhanced to a detectable level [29]. This demonstration has opened the way toward testing the astonishing predictions of the toroidal electrodynamics [37] and stimulated works on developing metamaterial and plasmonic systems exhibiting strong toroidal response [38-45].

In this paper, we aim to eliminate one of the major drawbacks of the toroidal metamaterial designs reported so far, namely, the dissipation loss. It results from the Ohmic resistance of the metallic resonators exploited by the existing designs and can substantially hinder the excitation of toroidal multipoles given their weak coupling to the external fields. To address this problem, we propose a completely nonmetallic (all-dielectric) metamaterial that is virtually free from dissipation and is capable of supporting strong resonant toroidal excitations in the terahertz part of the spectrum. We also show that owing to the unique topology of the toroidal dipolar mode, its electric field can be spatially confined within subwavelength externally accessible dielectric-free regions of the metamolecules, which makes the proposed metamaterial a viable platform for sensing or enhancement of light absorption.

\section{THE STRUCTURE OF THE SYSTEM}

Our dielectric metamaterial is based on clusters of subwavelength high-index dielectric cylinders. Each cluster contains four cylinders and represents the elementary building block of the metamaterial, i.e., its metamolecule [see Fig. 1(a)].

As of any dielectric structure, the electromagnetic response of such a metamolecule is underpinned by the displacement rather than conduction currents, which are induced in each cylinder by an incident electromagnetic

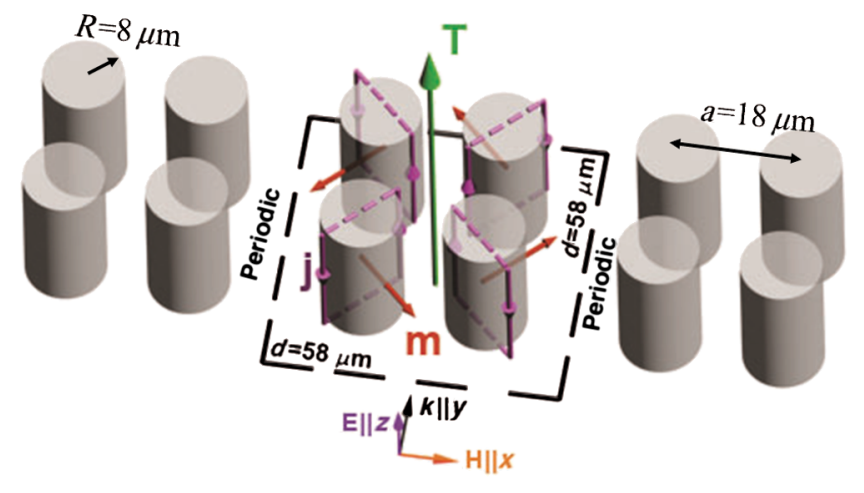

FIG. 1. A fragment of an all-dielectric metamaterial slab supporting toroidal dipolar excitation. Its metamolecule is composed of four closely spaced infinitely long high-index dielectric cylinders. The dashed box indicates the unit cell of the metamaterial, which is periodic along the $x$ axis only. Purple arrows show displacement currents $\mathbf{j}$ induced by the vertically polarized plane wave, red arrows show magnetic dipole moments of the constituent dielectric cylinders $\mathbf{m}$, and the green arrow represents the net toroidal dipole moment of the metamolecule $\mathbf{T}$.

wave. Depending on the radius and length of the cylinders, their dielectric constant, and the polarization of the incident wave, the electromagnetic scattering produced by the displacement currents can become resonant. It is this resonance (known as the Mie resonance [46]) that in the dielectric metamaterials is the counterpart of the lossy plasmonic resonance characteristic to the metallic metamolecules [47-52]. It was shown, in particular, that the Mie resonances of individual high-index dielectric cylinders could lead to a resonant magnetic dipolar response for orthogonal [53] as well as parallel [54] polarizations of the incident wave, thus rendering the dielectric metamaterials as a lossless solution for engineering the artificial magnetism [55].

The challenge of engineering toroidal response is to create a metamolecule that would support dynamically induced and spatially confined magnetization circulating along a loop. In our case, such metamolecule is formed by placing four dielectric cylinders close to each other, thereby promoting near-field coupling between the Mie-type magnetic modes excited individually in each cylinder. These modes correspond to the displacement currents $\mathbf{j}$ oscillating in the inward and outward parts of the cylinders in the opposite directions and are excited by the plane electromagnetic wave with an $\mathbf{E}$ vector parallel to the axes of the cylinders (see Fig. 1). We will show below that for a narrow range of frequencies, the magnetic moments of the modes $\mathbf{m}$, which oscillate perpendicular to the axes of the cylinders, may all become aligned head to tail, forming a dynamic vortex state with closed loops of the magnetic field confined well within the metamolecule. In an ideal case, such a state is characterized by zero net magnetic and electric multipole moments and a nonzero toroidal dipole moment oscillating along the axis of the metamolecule (see Fig. 1). 


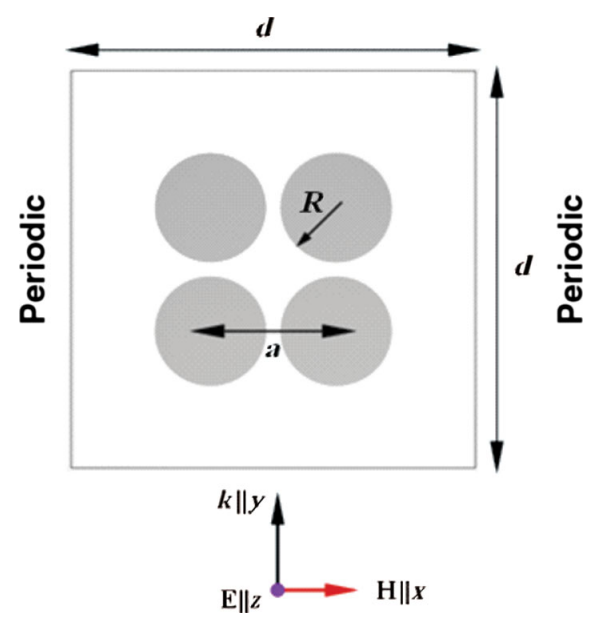

FIG. 2. Cross section of the dielectric metamolecule supporting toroidal dipolar excitation. The dimensions are $R=8 \mu \mathrm{m}$, $a=18 \mu \mathrm{m}$, and $d=58 \mu \mathrm{m}$.

\section{MATERIAL AND CURRENT-CHARGE DISTRIBUTION}

We assume in our model that the cylinders are made of $\mathrm{LiTaO}_{3}$, an ionic crystal that is known to exhibit strong polaritonic response at $\mathrm{THz}$ frequencies due to the excitation of optical phonons [48,56-58]. $\mathrm{LiTaO}_{3}$ cylinders can be practically realized using various methods of crystal growth [57,59]. The complex dielectric permittivity of $\mathrm{LiTaO}_{3}$ displays dispersion of the Lorenz type:

$$
\varepsilon=\varepsilon_{\infty} \frac{\omega^{2}-\omega_{L}^{2}+i \omega \gamma}{\omega^{2}-\omega_{T}^{2}+i \omega \gamma}
$$

where $\omega_{T} / 2 \pi=26.7 \mathrm{THz}$ is the frequency of the transverse optical phonons, $\omega_{L} / 2 \pi=46.9 \mathrm{THz}$ is the frequency of longitudinal optical phonons, $\gamma / 2 \pi=0.94 \mathrm{THz}$ is the damping factor due to dipole relaxation, and $\varepsilon_{\infty}=13.4$ is the limiting value of the permittivity for frequencies much higher than $\omega_{L}$. At frequencies well below the phonon resonances, the dielectric permittivity of $\mathrm{LiTaO}_{3}$ can be rather high, reaching $\varepsilon=41.4$. Importantly, at these frequencies, the crystal exhibits negligible dissipation loss, which makes it a standout candidate among other polaritonic materials. The $\mathrm{LiTaO}_{3}$ cylinders have the radius $R=8 \mu \mathrm{m}$ and are arranged inside the metamolecular cluster with the center-to-center separation $a=18 \mu \mathrm{m}$. The clusters are surrounded by vacuum or air and periodically placed along the $x$ axis with the period $d=58 \mu \mathrm{m}$, forming a one-metamolecule-thick metamaterial slab (structural thickness $58 \mu \mathrm{m}$ ). The cylinders are also assumed to be infinitely long, which allows us to describe the resulting structure using a two-dimensional model [see Fig. 2]. The electromagnetic properties of the metamaterial slab are computed with the aid of a commercial Maxwell's equation solver CST Microwave Studio using the standard modeling approach, where the metamaterial is represented by its single unit cell with properly applied boundary conditions (see Fig. 2).

The toroidal dipolar response is observed at around $1.89 \mathrm{THz}$ as a resonance of full transmission [see Fig. 4(a)]. The appearance of the toroidal excitation here is confirmed by calculating the distribution of the local fields and density of the displacement currents induced in the metamolecule, which we present in Fig. 3. The field maps clearly show a distinct vortex of the magnetic field that threads all four cylinders of the metamolecule and is produced by the displacement currents oscillating in the inward and outward parts of the cylinders in the opposite directions (poloidal current mode). Although the appearance of a magnetic vortex is also expected for an electric dipolar excitation (a)

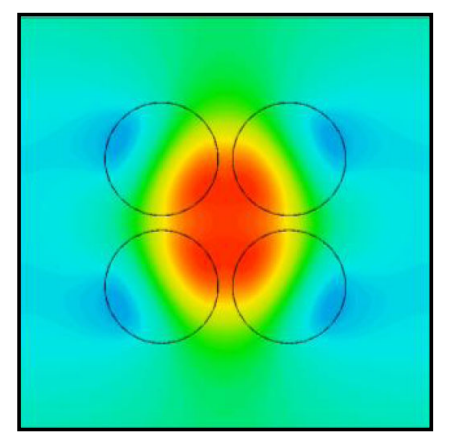

$\mathbf{E}_{z}$

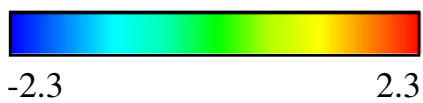

(b)

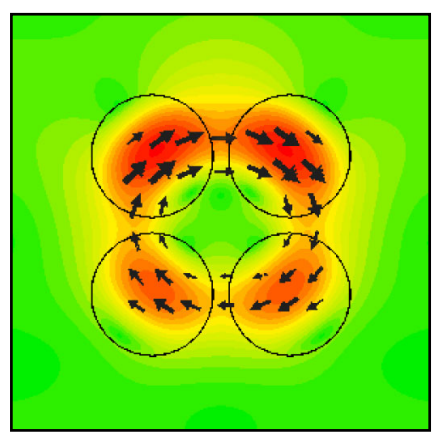

$|\mathbf{H}|$

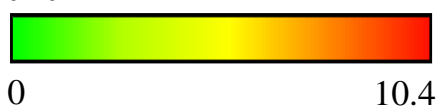

(c)

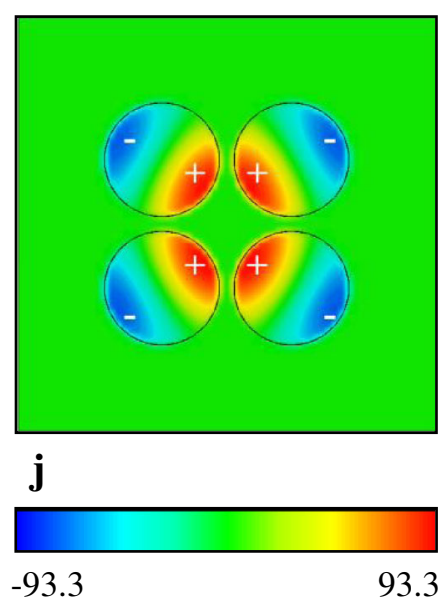

FIG. 3. (a)-(c) Calculated distributions of the corresponding electric field ( $z$ component $\mathbf{E}_{z}$ ), magnetic field (absolute value $\left.|\mathbf{H}|\right)$, and amplitude of the displacement current $\mathbf{j}$ ( $z$ component) induced in the metamolecule at $1.89 \mathrm{THz}$. Arrows show instantaneous directions in the magnetic-field distribution. 
(a)

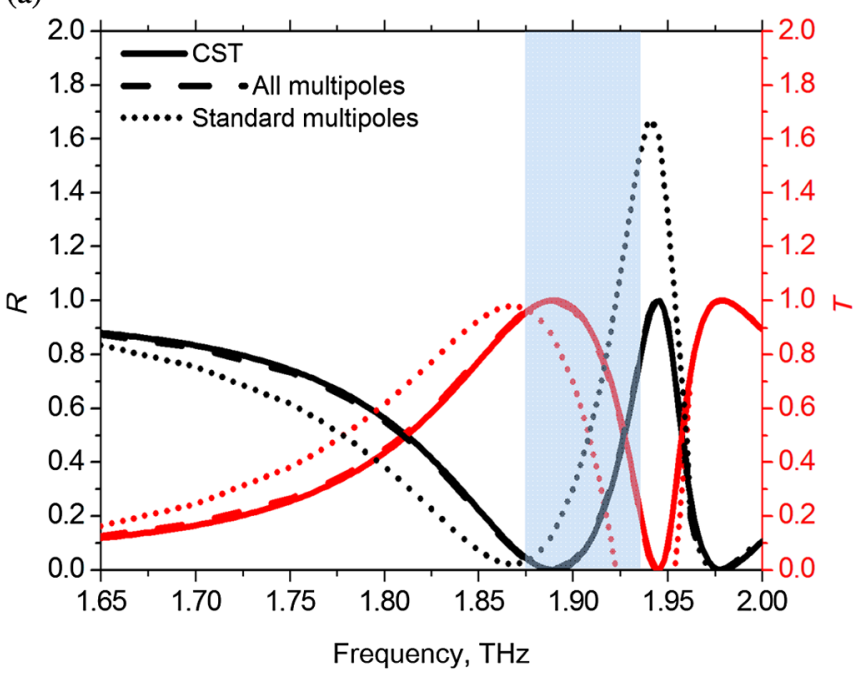

(b)

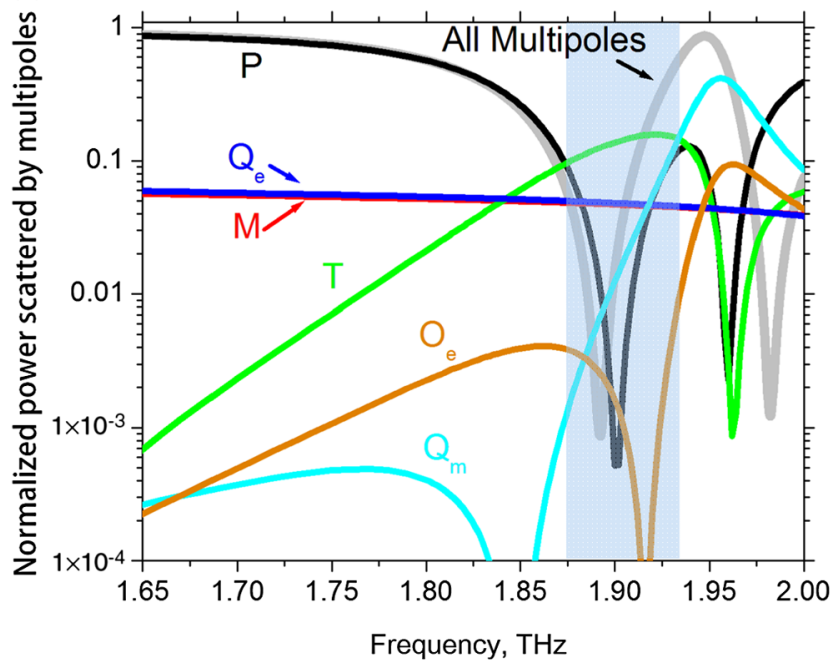

FIG. 4. (a) Transmission $T$ (red lines) and reflection $R$ (black lines) spectra calculated for the metamaterial slab composed of fourcylinder clusters periodically placed along the $x$ axis, as shown in Fig. 2. Solid curves correspond to the results of the CST Microwave Studio simulations. The dashed curves are obtained based on the multipole decomposition of the displacement current data, which includes both the standard multipole terms (up to the magnetic octupole) and toroidal multipoles (up to the toroidal quadrupole). The dotted curves are obtained based on the multipole decomposition missing the toroidal multipoles. (b) Contributions of the six strongest multipolar excitations to the reflection of the metamaterial array. The gray curve marked "All multipoles" gives the normalized total power scattered by the multipoles and corresponds to the reflection of the metamaterial slab, shown by the dashed black curve in (a). It is obtained by coherent (amplitude and phase) summation of all multipole contributions. The log scale in the $y$ axis in (b) is chosen so as to reveal more clearly the contribution of the quadrupole terms as well.

(think of magnetic-field lines circling around an electrical current), the induced magnetic field in the present case is confined within a well-defined ringlike region ("torus"). Such spatial localization of the magnetic field and the corresponding poloidal current mode are the key signatures of the toroidal dipolar excitation. The slight asymmetry in the distribution of the magnetic or electric field and displacement currents is attributed to the presence of other multipolar excitations, most notably, the electric quadrupole and magnetic dipole. The latter provides here the second and third strongest contributions, as will become evident below. Correspondingly, the asymmetry of the magnetic vortex results from the superposition of toroidal and magnetic dipolar modes, while the asymmetry of the displacement currents (electric field) results from the superposition of the toroidal dipole and electric quadrupole.

To assess the role of the toroidal excitation in forming the observed resonant response, we compare the relative strength [60] of the toroidal dipole and standard chargecurrent multipoles in terms of the electromagnetic power they scatter in the far-field zone. The multipole moments induced in the metamolecules are calculated based on the density of the displacement currents, i.e., currents resulted from the displacement of charges in the dielectric. The latter are extracted from the simulated near-field distribution within the dielectric cylinder data using the following formula [61]:

$$
\mathbf{j}=\frac{d \mathbf{P}}{d t}=\varepsilon_{0}(\varepsilon-1) \frac{d \mathbf{E}}{d t}=i \omega \varepsilon_{0}(\varepsilon-1) \mathbf{E} .
$$

This approach allows us to unambiguously link the nearfield signature of the multipolar charge-current excitations to their electromagnetic response in the far field. The latter would not be possible with the multipole expansion based on radiated fields (as featured in the Mie-scattering approach) since the same-order toroidal and electric multipoles emit radiation with the same angular momentum and parity properties and thus appear indistinguishable to any distant observer [12]. Moreover, in the case of a metamaterial array, the scattered fields propagate into the far-field zone as plane waves, and, therefore, their expansion into a series of spherical harmonics using the Mie-type analysis would provide no information on the types of the elementary excitations supported by individual metamolecules.

For each type of multipole, the far-field scattering is characterized by electromagnetic radiation reemitted by the entire array of the corresponding multipoles and hence taking the form of plane waves [60]. We proceed by first extruding the simulated 2D current distribution to a small but finite extent along the $z$ axis. The resulting 3D current distribution is then used to compute the multipole moments of the metamolecule per its unit length, while the radiation patterns of the corresponding 
multipoles are described through the standard spherical vector harmonics. The metamolecules are assumed to be excited all in phases, as is expected in the case of planewave illumination at normal incidence. The field radiated by the array of multipoles is calculated at a point in the far-field zone by summing the multipolar scattering of each type from all the metamolecules along the $x$ and $z$ axes (see Ref. [60] for more details). The results of such calculations are presented in Fig. 4(b), which shows the power radiated by the arrays of the induced toroidal dipole $\mathbf{T}$ and the five strongest multipoles, namely, the electric dipole $\mathbf{P}$, magnetic dipole $\mathbf{M}$, electric quadrupole $\mathbf{Q}_{\mathrm{e}}$, magnetic quadrupole $\mathbf{Q}_{\mathrm{m}}$, and electric octupole $\mathbf{O}_{\mathrm{e}}$, normalized to the incident power. One can see that at the resonance, the contribution of the electric dipole is strongly suppressed and there is a narrow range of frequencies, from 1.87 to $1.93 \mathrm{THz}$ (shown by the gray box in Fig. 4) where the far-field scattering due to the resonant toroidal excitation dominates all other standard multipoles. In particular, the power scattered through the toroidal dipole moment in this frequency range is more than 2 times higher than that of the magnetic dipole and electric quadrupole moments. The key role of the toroidal excitation can be further demonstrated by calculating the reflection $R$ and transmission $T$ of the metamaterial slab using the obtained multipole scattering data. In particular, reflection is given by the coherent sum of all multipole fields (including amplitude and phase) scattered by the metamolecules in the backward direction; transmission is generally given by the coherent superposition of the incident and forward-scattered fields, but in the absence of losses (our case), it can be calculated simply as $T=1-R$. We stress from Fig. 4(a) that the metamaterial response can be exactly reproduced by taking into account all the multipole contributions, while ignoring the toroidal dipole moment makes the correct replication of the observed resonance impossible. Most notably, the relative error of replicating the spectrum based on the standard charge-current multipoles only is diverging at the frequency of the toroidal resonance $1.89 \mathrm{THz}$. Furthermore, failure to account for the toroidal dipole moment leads to simply unphysical behavior of the metamaterial response near $1.95 \mathrm{THz}$ where its transmission exceeds 100\% [see the dotted curve in Fig. 4(a)]. At higher frequencies, though, the discrepancy in the calculated spectra appears to be much smaller, which is the result of the toroidal dipole scattering contribution becoming negligible.

We note in passing that the resonant excitation of the toroidal dipole mode results from the coupling of the magnetic Mie modes of the four cylinders comprising our metamolecule. The frequency of the first "magnetic" mode of a single dielectric cylinder, which in our case is $2.18 \mathrm{THz}$, can be estimated analytically by setting to 0 the denominator of the Mie-scattering coefficient [62]

$$
\begin{aligned}
& b_{1}=\frac{n J_{1}^{\prime}\left(z_{2}\right) J_{1}\left(z_{1}\right)-J_{1}\left(z_{2}\right) J_{1}^{\prime}\left(z_{1}\right)}{n J_{1}^{\prime}\left(z_{2}\right) H_{1}^{(1)}\left(z_{1}\right)-J_{1}\left(z_{2}\right) H_{1}^{(1)^{\prime}}\left(z_{1}\right)}, \\
& z_{2}=k_{2} R, \quad z_{1}=k_{0} R
\end{aligned}
$$

where $k_{0}=\omega / c$ is the wave vector in free space, $k_{2}=k_{0} \sqrt{\varepsilon}$, $n=\sqrt{\varepsilon}$, and $J_{1}$ and $H_{1}{ }^{(1)}$ are the Bessel function and the first-kind Hankel function of the first order, respectively. The lowering of the toroidal resonance frequency compared to the Mie-mode frequency is the direct consequence of the near-field coupling between the magnetic Mie modes induced in each cylinder, which in the case of the circular head-to-tail dipole arrangement (see Fig. 1) reduces the energy of the resulting toroidal mode. It can be understood using a very crude model, where the magnetic dipolar mode in each cylinder is represented by a pair of antiparallel steady currents of equal strength separated by distance $2 R$. Excitation of the toroidal mode in a cluster of four cylinders then corresponds to four pairs of antiparallel currents separated by distance $a$. The potential energies of such systems of two currents $w$ and eight currents $W$ can be readily calculated based on the Biot-Savart law. The binding energy of the toroidal mode is then given by $\Delta W=W-4 w$ and corresponds to $6 \%$ of $w$ (energy of the magnetic mode in isolation), which, given the simplicity of the model, agrees well with the outcome of our simulations.

Remarkably, the mode's electric field has only the longitudinal component (parallel to the axis of the cylinders) and is concentrated in the central part of the metamolecule in a spot smaller that $\lambda / 5$ [see Fig. 2]. Such localization of the electric field is characteristic only to the toroidal dipolar mode, where the electric field is laterally confined within the eye of the subwavelength magnetic-field vortex and is sustained there by curl $\mathbf{H}$ in the absence of polarization or charge-density oscillations. As a result, the field distribution is seen to reach its maximum in the dielectric-free region of the metamolecule rendering it as a subwavelength externally accessible cavity that can be exploited, for example, in sensing or achieving stronger optical absorption. In particular, such a cavity may be seen as a part of a microfluidic system designed for high-throughput biological screening or biomedical and environmental monitoring in the THz part of the spectrum. Sensing applications will benefit from the unique property of the toroidal excitation, where loading the hot-spot areas with a test sample will only affect the parameters of the toroidal resonance (amplitude, frequency, and phase) making the unaffected neighboring higher-frequency resonance a proper reference feature in the spectrum.

Apart from engaging the toroidal dipolar mode, the localization of the electric field outside the dielectric region can be achieved in the gap between two transversally polarized cylinders. In this case, however, the externally applied electric field must be nonresonant (quasistatic), since the fields of the resonantly induced Mie modes are 
localized inside the cylinders. Such a capacitorlike configuration provides confinement of the electric field in only one dimension, while obtaining large field enhancement in the gap would require dielectrics with the permittivity substantially higher than 41.4.

Finally, we would like to comment on the mechanism of the metamaterial's electromagnetic transparency observed near the toroidal resonance [Fig. 4(a)]. It results from the interference of the fields scattered by nonresonant magnetic dipolar and electric quadrupolar modes and by the resonant toroidal dipolar mode [see Fig. 4(b)]. Although the angular distributions of the multipolar scattering are anisotropic and normally do not match for the individual multipoles of different types (except for the electric and toroidal multipoles $[11,13]$ ), the omnidirectional interference of the fields scattered by $\mathbf{T}, \mathbf{M}$, and $\mathbf{Q}_{\mathbf{e}}$ is nevertheless possible for the arrays of these multipoles arranged on a regular grid with subwavelength periodicity (as it happens in the metamaterial slab). Indeed, the emission diagrams of such multipole arrays collapse around the normal to their plane, signifying the regime of plane-wave scattering with only two scattering directions available-forward and backward. In our case, the plane waves reemitted by the combination of induced magnetic dipoles and electric quadrupoles oscillate in phase but with a $\pi$ delay compared to the waves produced by the toroidal dipoles. It makes their far-field interference destructive in the backward direction, which is complete at around $1.89 \mathrm{THz}$.

Because of the generally incoherent nature of the metamaterial response, the results of our study can be readily extended to the case of a finite-size slab. We also confirm this fact by setting the length of the rods in our model to a realistic value. Our simulations show that the electromagnetic scattering at the ends of the dielectric rods would not significantly affect the relative strength of the toroidal excitations, and the overall response of the metamaterial slab would persist (see Appendix A 3 for more details).

In conclusion, we proposed and theoretically studied a novel class of all-dielectric metamaterials that exhibit a resonant toroidal dipolar response in the $\mathrm{THz}$ part of the spectrum. Our metamaterials are based on subwavelength clusters of high-index dielectric cylinders operating in the regime of resonant Mie scattering. We show that the near-field coupling between the individual Mie modes of the cylinders is capable of suppressing all standard multipoles and make electromagnetic scattering due to the resulting toroidal dipolar excitations the dominant mechanism of the metamaterial response. Our findings thus indicate that the notion of the toroidal dipole can be crucial for the correct interpretation of electromagnetic properties in artificially structured material systems. The proposed metamaterials can be readily fabricated from low-loss polaritonic material $\mathrm{LiTaO}_{3}$ and, given the unique topology of the toroidal dipolar mode, may be employed as a platform for sensing or enhancement of light absorption.

\section{ACKNOWLEDGMENTS}

The authors acknowledge the financial support by the EU projects ENSEMBLE and By-Nanoera and the Greek project ERC-02 ExEL (Grant No. 6260). Work at Ames Laboratory was partially supported by the Department of Energy (Basic Energy Sciences) under Contract No. DEAC02-07CH11358 (computational studies). Work at the National Research University "Moscow Power Engineering Institute" was supported by the Russian Foundation for Basic Research (Grant Agreements No. 13-02-00732 and No. 13-08-01278) and the Russian Federal Program "Scientific and ScientificPedagogical Staff of Innovative Russia" for 2009-2013 (Agreement No. 14.B37.21.1211). V. A. F., V. S., and N. I.Z. acknowledge financial support of the Royal Society and the U.K.'s Engineering and Physical Sciences Research Council under the Programme Grant on Photonic Metamaterials and Career Acceleration Fellowship (V. A. F.). Work at Nanyang Technological University was partially supported by the MOE Singapore Grant No. MOE2011-T3-1-005.

\section{APPENDIX}

\section{A. Toroidal response versus incidence angle and polarization}

We also characterize the spectral response of the proposed dielectric metamaterial with respect to the angle of incidence. Figure 5(a) shows the results of our simulations for the case of TE polarization where the electric vector of the plane incident wave is kept parallel to the axes of the cylinders. Evidently, the toroidal resonance can be observed at oblique incidence, and it is seen to shift to higher frequencies with increasing angle due to spatial dispersion of the metamaterial slab onto the not-extreme subwavelength size of the unit cell. The persistence of the toroidal mode is also confirmed by calculating the corresponding magnetic near-field distribution, as well as by comparing its contribution to the far-field scattering with that of the leading standard dipoles [see Figs. 5(b) and 5(c)]. Although at large angles of incidence the ringlike pattern of the mode's magnetic field appears to be disturbed (due to the modified interference between the toroidal mode and the incident field), it still features a pronounced antisymmetric component that ensures a strong toroidal dipolar response for each metamolecule [see Fig. 5(c)].

It is not possible to observe the toroidal dipolar response for the orthogonal polarization, i.e., when the magnetic vector of the plane wave has been set parallel to 
(a)

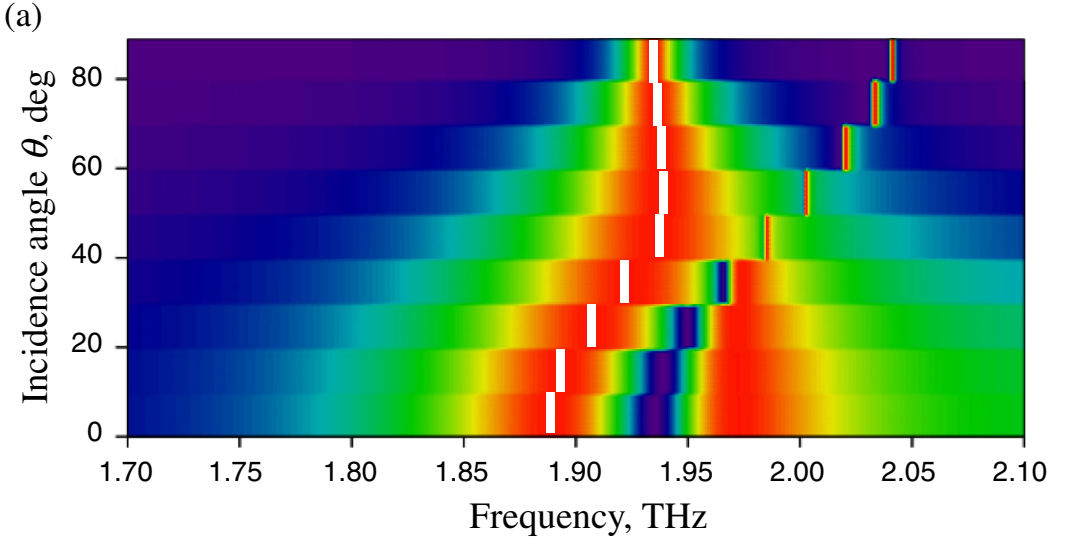

(b)

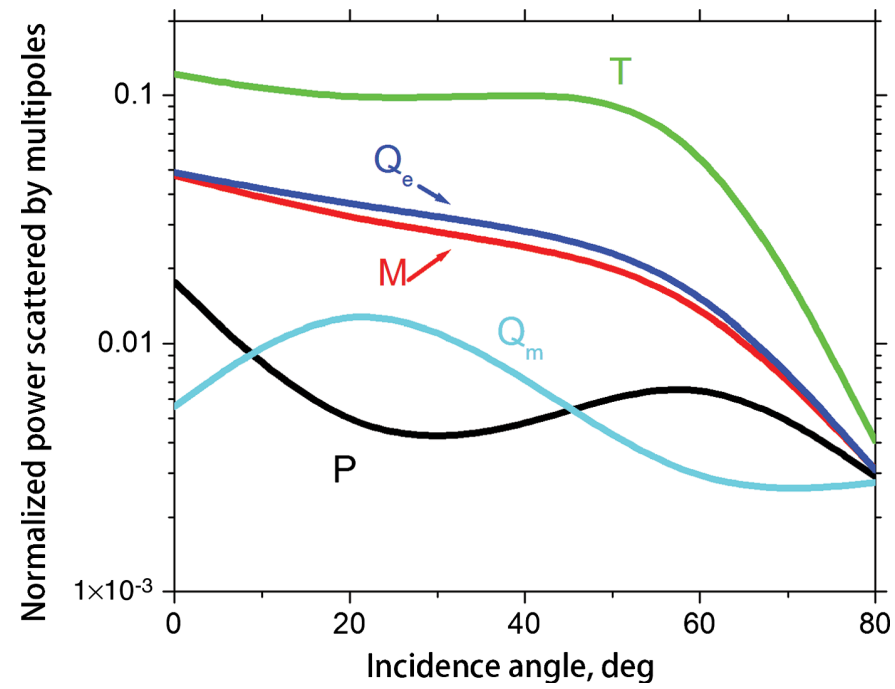

(c)

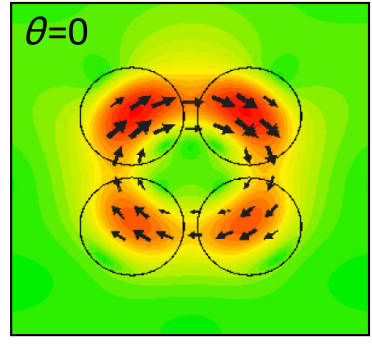

$|\mathbf{H}|$

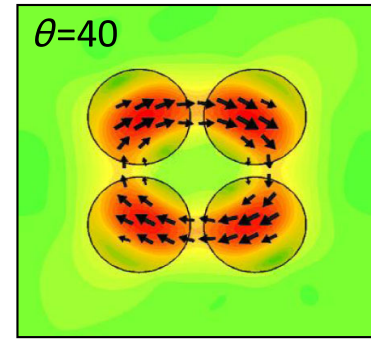

$|\mathbf{H}|$

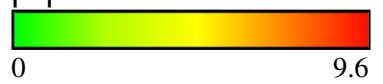

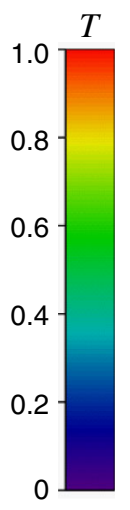

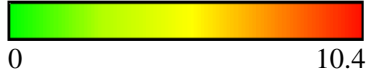

FIG. 5. (a) Transmission $T$ spectra of the metamaterial slab calculated with the CST Microwave Studio for obliquely incident $E$-polarized plane waves (the electric vector of the waves is set parallel to the axes of the cylinders) with the step of 10 degrees. The white bars mark the frequency of the toroidal mode. (b) Relative strength of four leading standard multiples and a toroidal dipole compared for various angles of incidence at the frequency of the toroidal mode. (c) Magnetic near-field distributions calculated for angles of incidence $\theta=0^{\circ}, 40^{\circ}, 80^{\circ}$ at the frequency of the toroidal mode [corresponding to the white bars in (a)].

the axes of the cylinders (Fig. 6). Although such polarization is shown to induce magnetization in a dielectric cylinder [53], the corresponding magnetic dipole moment is oriented parallel to the axis of the cylinder. In a cluster of four infinitely long cylinders, such an orientation cannot lead to the formation of a magnetic-field vortex characteristic to the toroidal mode (see the insets to Fig. 6). 


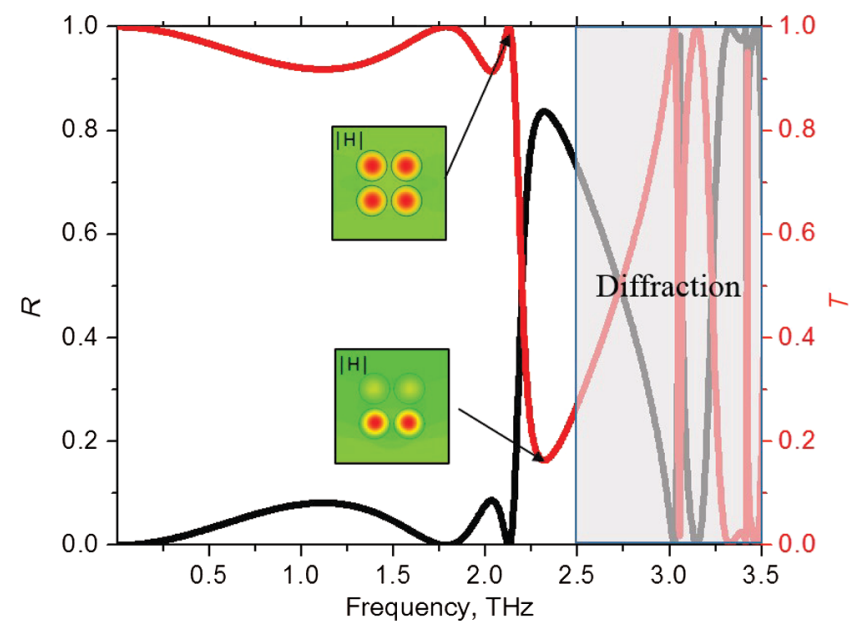

FIG. 6. Transmission $T$ (red line) and reflection $R$ (black line) spectra of the metamaterial slab calculated with the CST Microwave Studio for normally incident $\mathbf{H}$-polarized plane waves. (The magnetic vector of the waves is set parallel to the axes of the cylinders.) The insets show the magnetic near-field distributions calculated at 2.13 and $2.32 \mathrm{THz}$.

\section{B. Higher multipole contribution close to the toroidal resonance}

We note in passing that the metamaterial response to normally incident plane waves also features a very strong contribution from magnetic quadrupole $\mathbf{Q}_{\mathbf{m}}$ near $1.95 \mathrm{THz}$ [Fig. 4(b)]. There are two main reasons. First, the dielectric cluster, although subwavelength, is relatively large $(\lambda / 5)$ and the constituent cylinders have very a large dielectric constant, which should lead to large retardation in the interaction between the charges induced in each cylinder. It is therefore not unreasonable to expect that there will be a substantial contribution from multipoles higher than the dipole. Second, due to a particular symmetry of the metamolecule, the excitation of certain multipoles must become more favorable than others. In particular, the antisymmetric current modes excited in each cylinder at around 1.90 and $1.95 \mathrm{GHz}$ naturally make the electric dipole moment vanishing (hence, it scatters at the level of approximately 0.001), while the fourfold-symmetric spatial arrangement of the antisymmetric modes leads to substantial net toroidal dipole, magnetic quadrupole, and electric octupole moments. As in the case of toroidal dipolar excitation, the magnetic quadrupole emerges as the result of the coupling of individual magnetic dipole modes induced in each of four cylinders (see Fig. 7). Contrary to the toroidal dipolar mode, the electric field of the magnetic quadrupolar excitation is completely expelled from the central region of the metamolecule. It presents an intriguing opportunity of using the metamaterial as a microscopic cloaking device, where the presence of an object placed in the center of the metamolecule may not be revealed.

\section{Toroidal metamolecules made of finite cylinders}

In a real experiment, the metamaterial slab will have a finite size, and it is therefore important to understand how its response will deviate from that of an infinitely sized slab. For that, we model the transmission and reflection of a metamaterial slab with the length of its rods set to $400 \mu \mathrm{m}$. Evidently, the calculated metamaterial spectra [see Fig. 8(a)] acquire few additional small spectral features, as well as demonstrate a slight redshift and reduction of the reflection and transmission levels, but generally retain the same profile as in the $2 \mathrm{D}$ case [see Fig. 4(a)]. As for the multipole contributions [see Fig. 8(b)], although the spectral dependences of the scattered power appear to have changed for most of the multipoles, the toroidal dipole still provides the (a)

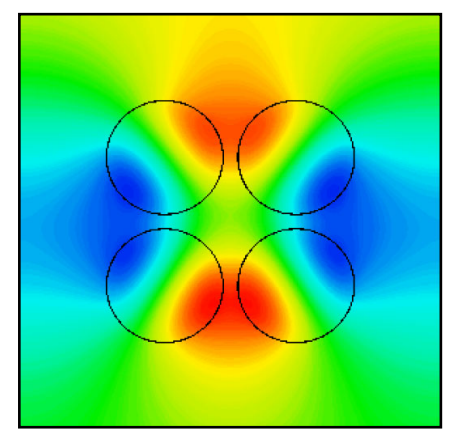

$\mathbf{E}_{z}$

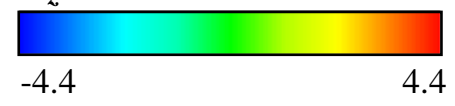

(b)

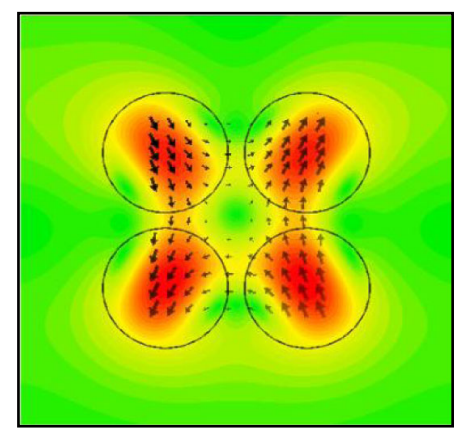

|H|

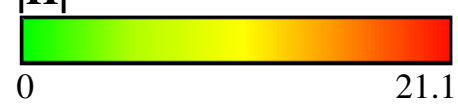

(c)

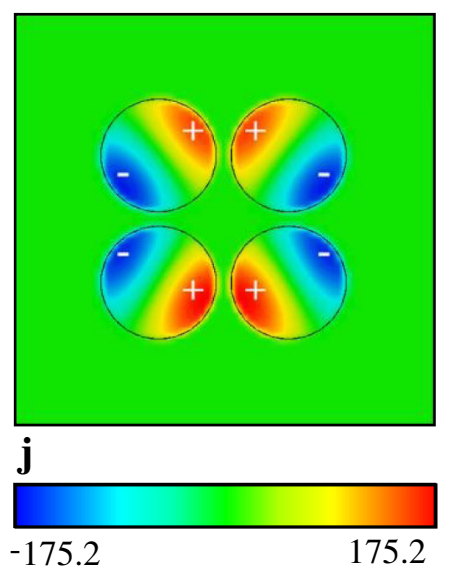

FIG. 7. (a),(b) Calculated distributions of the absolute values of the (a) electric field ( $z$ component) and (b) magnetic field induced in the metamolecule at $1.95 \mathrm{THz}$. (c) The distribution of the displacement current $\mathbf{j}$ within the dielectric rods corresponds to the magnetic quadrupole response induced in the metamolecule. 
(a)

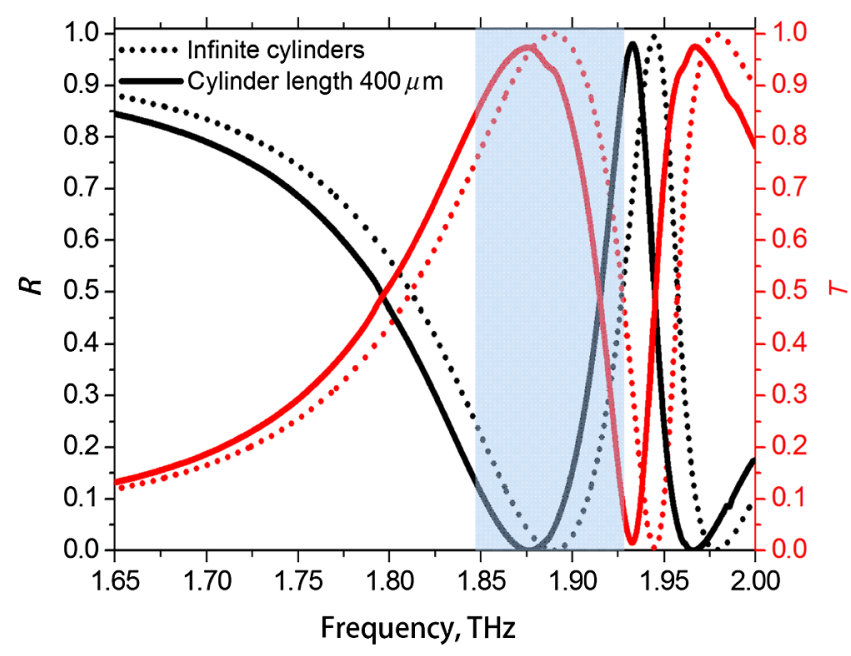

(b)

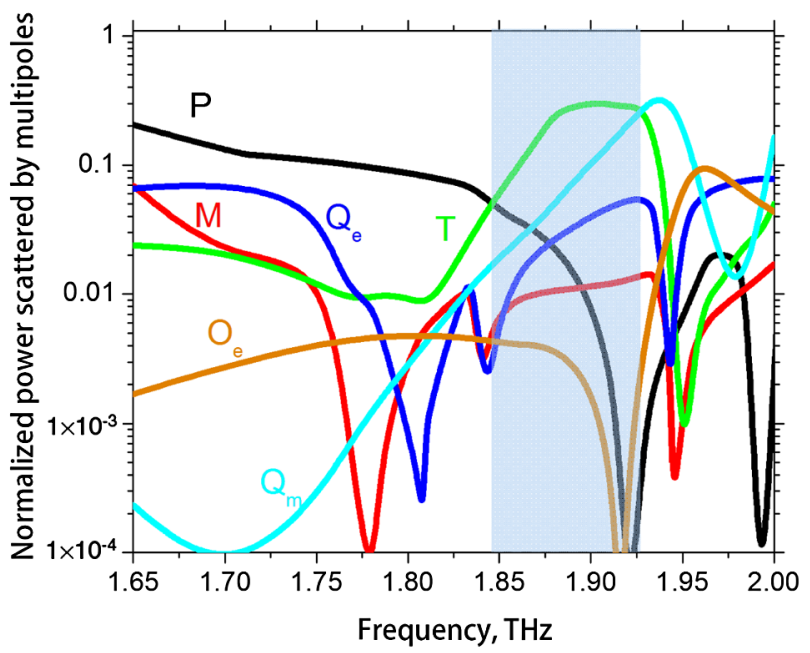

FIG. 8. (a) Transmission $T$ (red lines) and reflection $R$ (black lines) spectra calculated for the metamaterial slab composed of four-cylinder clusters periodically placed as described in the text. The solid curves correspond to the results of the CST Microwave Studio simulations for $400-\mu \mathrm{m}$-in-height cylinders. The dashed curves are obtained for the case of infinite-in-height cylinders. (b) Individual contributions of the six strongest multipolar excitations to the normalized power radiated (scattered) by our metamaterial array of finite-height cylinder clusters near its toroidal resonance.

(a)

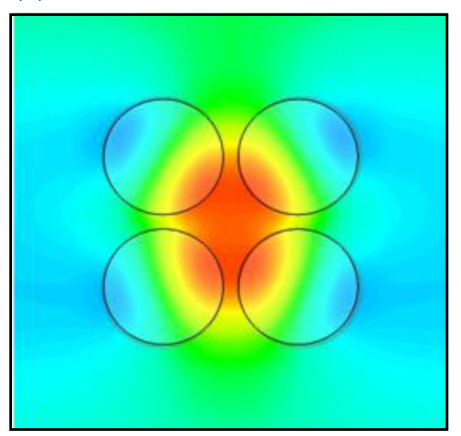

$\mathbf{E}_{z}$

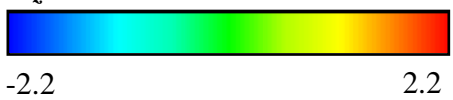

(b)

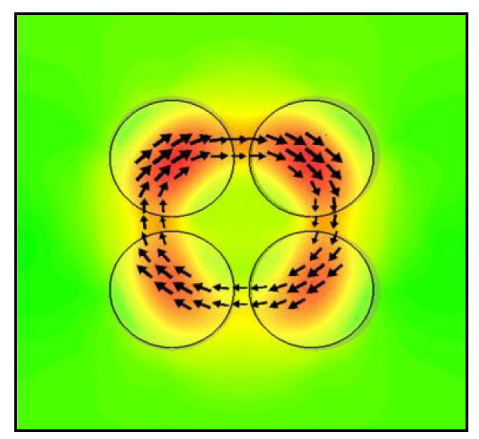

$|\mathbf{H}|$

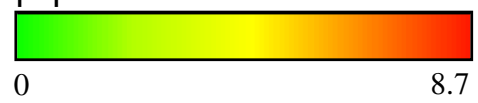

(c)

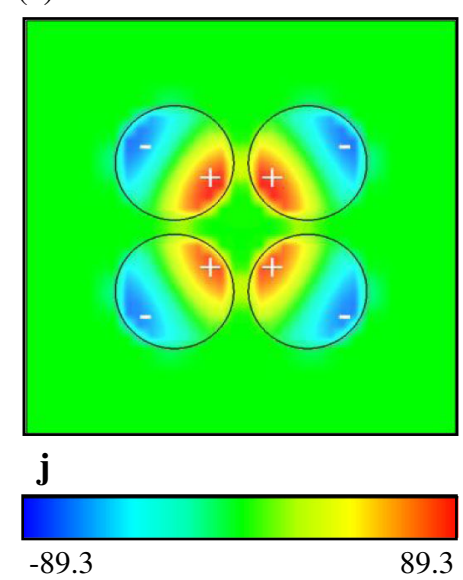

FIG. 9. (a)-(c) Calculated distributions of the corresponding electric field ( $z$ component $\mathbf{E}_{z}$ ), magnetic field (absolute value $|\mathbf{H}|$ ), and amplitude of the displacement current $\mathbf{j}$ induced in the metamolecule at $1.87 \mathrm{THz}$. The arrows show instantaneous directions in the magnetic-field distribution.

dominant contribution at the reflection minimum near 1.87 THz. The strong presence of the toroidal mode here is also confirmed by calculating the distributions of electric and magnetic fields and displacement currents [see Figs. 9(a)-9(c)].

[1] Y. B. Zel'dovich, Electromagnetic Interaction with Parity Violation, Sov. Phys. JETP 6, 1184 (1958).

[2] I. I. Naumov L. Bellaiche, and H. X. Fu, Unusual Phase Transitions in Ferroelectric Nanodisks and Nanorods, Nature (London) 432, 737 (2004).
[3] M. Kläui, C. A. F. Vaz, L. Lopez-Diaz, and J. A. C. Bland, Vortex Formation in Narrow Ferromagnetic Rings, J. Phys. Condens. Matter 15, R985 (2003).

[4] Y. F. Popov, A. M. Kadomtseva, G. P. Vorob'ev, V. A. Timofeeva, D. M. Ustinin, A. K. Zvezdin, and M. M. Tegeranchi, Magnetoelectric Effect and Toroidal Ordering in $\mathrm{Ga}_{2-x} \mathrm{Fe}_{x} \mathrm{O}_{3}$, J. Exp. Theor. Phys. 87, 146 (1998).

[5] Y. F. Popov, A. M. Kadomtseva, D. V. Belov, G. P. Vorob'ev, and A. K. Zvezdin, Magnetic-Field-Induced Toroidal Moment in the Magnetoelectric $\mathrm{Cr}_{2} \mathrm{O}_{3}$, JETP Lett. 69 , 330 (1999).

[6] Y. V. Kopaev, Toroidal Ordering in Crystals, Phys. Usp. 52, 1111 (2009). 
[7] A. K. Zvezdin, V. V. Kostyuchenko, A. I. Popov, A. F. Popkov, and A. Ceulemans, Toroidal Moment in the Molecular Magnet $V_{15}$, Phys. Rev. B 80, 172404 (2009).

[8] L. Ungur, S. K. Langley, T. N. Hooper, B. Moubaraki, E. K. Brechin, K. S. Murray, and L. F. Chibotaru, Net Toroidal Magnetic Moment in the Ground State of a $\{D y-6\}-$ Triethanolamine Ring, J. Am. Chem. Soc. 134, 18554 (2012).

[9] A. Ceulemans, L. F. Chibotaru, and P. W. Fowler, Molecular Anapole Moments, Phys. Rev. Lett. 80, 1861 (1998).

[10] V. M. Dubovik and V. V. Tugushev, Toroid Moments in Electrodynamics and Solid-State Physics, Phys. Rep. 187, 145 (1990).

[11] E. E. Radescu and G. Vaman, Exact Calculation of the Angular Momentum Loss, Recoil Force, and Radiation Intensity for an Arbitrary Source in Terms of Electric, Magnetic, and Toroid Multipoles, Phys. Rev. E 65, 046609 (2002).

[12] G. N. Afanasiev and Y. P. Stepanovsky, The ElectromagneticField of Elementary Time-Dependent Toroidal Sources, J. Phys. A 28, 4565 (1995).

[13] A. T. Góngora, and E. Ley-Koo, Complete Electromagnetic Multipole Expansion Including Toroidal Moments, Rev. Mex. Fis. E52, 188 (2006).

[14] G. N. Afanasiev, Simplest Sources of Electromagnetic Fields as a Tool for Testing the Reciprocity-like Theorems, J. Phys. D 34, 539 (2001).

[15] K. Sawada and N. Nagaosa, Optical Magnetoelectric Effect in Multiferroic Materials: Evidence for a Lorentz Force Acting on a Ray of Light, Phys. Rev. Lett. 95, 237402 (2005).

[16] S. A. Maier and H. A. Atwater, Plasmonics: Localization and Guiding of Electromagnetic Energy in Metal/Dielectric Structures, J. Appl. Phys. 98, 011101 (2005).

[17] K. A. Willets and R. P. Van Duyne, in Annual Review of Physical Chemistry (Annual Reviews, Palo Alto, 2007), p. 267-297.

[18] S. Lal, S. Link, and N. J. Halas, Nano-optics from Sensing to Waveguiding, Nat. Photonics 1, 641 (2007).

[19] M. A. Noginov, G. Zhu, A. M. Belgrave, R. Bakker, V. M. Shalaev, E. E. Narimanov, S. Stout, E. Herz, T. Suteewong, and U. Wiesner, Demonstration of a Spaser-Based Nanolaser, Nature (London) 460, 1110 (2009).

[20] H. Wang, E. C. Y. Yan, E. Borguet, and K. B. Eisenthal, Second Harmonic Generation from the Surface of Centrosymmetric Particles in Bulk Solution, Chem. Phys. Lett. 259, 15 (1996).

[21] J. Shan, J. I. Dadap, I. Stiopkin, G. A. Reider, and T. F. Heinz, Experimental Study of Optical Second-Harmonic Scattering from Spherical Nanoparticles, Phys. Rev. A 73, 023819 (2006).

[22] F. Wise, L. J. Qian, and X. A. Liu, Applications of Cascaded Quadratic Nonlinearities to Femtosecond Pulse Generation, J. Nonlinear Opt. Phys. Mater. 11, 317 (2002).

[23] S. Kujala, B. K. Canfield, M. Kauranen, Y. Svirko, and J. Turunen, Multipolar Analysis of Second-Harmonic Radiation from Gold Nanoparticles, Opt. Express 16, 17196 (2008).

[24] M. Zdanowicz, S. Kujala, H. Husu, and M. Kauranen, Effective Medium Multipolar Tensor Analysis of
Second-Harmonic Generation from Metal Nanoparticles, New J. Phys. 13, 023025 (2011).

[25] N. V. Hud and I. D. Vilfan, in Annual Review of Biophysics and Biomolecular Structure (Annual Reviews, Palo Alto, 2005), p. 295-318.

[26] M. M. Hingorani and M. O'Donnell, A Tale of Toroids in DNA Metabolism, Nat. Rev. Mol. Cell Biol. 1, 22 (2000).

[27] A. A. Simpson, Y.Z. Tao, P. G. Leiman, M. O. Badasso, Y. N. He, P. J. Jardine, N. H. Olson, M. C. Morais, S. Grimes, D. L. Anderson, T. S. Baker, and M. G. Rossmann, Structure of the Bacteriophage Phi 29 DNA Packaging Motor, Nature (London) 408, 745 (2000).

[28] V. A. Fedotov, K. Marinov, A. D. Boardman, and N. I. Zheludev, On the Aromagnetism and Anapole Moment of Anthracene Nanocrystals, New J. Phys. 9, 95 (2007).

[29] T. Kaelberer, V. A. Fedotov, N. Papasimakis, D. P. Tsai, and N. I. Zheludev, Toroidal Dipolar Response in a Metamaterial, Science 330, 1510 (2010).

[30] V. G. Veselago, The Electrodynamics of Substances with Simultaneously Negative Values of $\varepsilon$ and $\mu$, Sov. Phys. Usp. 10, 509 (1968).

[31] J. B. Pendry, Negative Refraction Makes a Perfect Lens, Phys. Rev. Lett. 85, 3966 (2000).

[32] N. I. Zheludev, The Road Ahead for Metamaterials, Science 328, 582 (2010).

[33] C. M. Soukoulis and M. Wegener, Optical MetamaterialsMore Bulky and Less Lossy, Science 330, 1633 (2010).

[34] C. M. Soukoulis and M. Wegener, Past Achievements and Future Challenges in the Development of ThreeDimensional Photonic Metamaterials, Nat. Photonics 5, 523 (2011).

[35] A. Boltasseva and H. A. Atwater, Low-Loss Plasmonic Metamaterials, Science 331, 290 (2011).

[36] Y. Liu and X. Zhang, Metamaterials: A New Frontier of Science and Technology, Chem. Soc. Rev. 40, 2494 (2011).

[37] V. A. Fedotov, A. V. Rogacheva, V. Savinov, D. P. Tsai, and N. I. Zheludev, Resonant Transparency and Non-trivial Non-radiating Excitations in Toroidal Metamaterials, Sci. Rep. 3, 2967 (2013).

[38] Z.-G. Dong, P. Ni, J. Zhu, X. Yin, and X. Zhang, Toroidal Dipole Response in a Multifold Double-Ring Metamaterial, Opt. Express 20, 13065 (2012).

[39] L. Y. Guo, M. H. Li, Q. W. Ye, B. X. Xiao, and H. L. Yang, Electric Toroidal Dipole Response in Split-Ring Resonator Metamaterials, Eur. Phys. J. B 85, 1 (2012).

[40] Y. Fan, Z. Wei, H. Li, H. Chen, and C. M. Soukoulis, Low-Loss and High-Q Planar Metamaterial with Toroidal Moment, Phys. Rev. B 87, 115417 (2013).

[41] Y.-W. Huang, W. T. Chen, P. C. Wu, V. Fedotov, V. Savinov, Y.Z. Ho, Y.-F. Chau, N. I. Zheludev, and D. P. Tsai, Design of Plasmonic Toroidal Metamaterials at Optical Frequencies, Opt. Express 20, 1760 (2012).

[42] B. Ögüt, N. Talebi, R. Vogelgesang, W. Sigle, and P. A. van Aken, Toroidal Plasmonic Eigenmodes in Oligomer Nanocavities for the Visible, Nano Lett. 12, 5239 (2012).

[43] Z. G. Dong, J. Zhu, J. Rho, J. Q. Li, C. G. Lu, X. B. Yin, and X. Zhang, Optical Toroidal Dipolar Response by an 
Asymmetric Double-Bar Metamaterial, Appl. Phys. Lett. 101, 144105 (2012).

[44] W. T. C. Yao-Wei Huang, P.C. Wu, V. A. Fedotov, N. I. Zheludev, and D. P. Tsai, Toroidal Lasing Spaser, Sci. Rep. 3, 1237 (2013).

[45] Z. G. Dong, J. Zhu, X. B. Yin, J. Q. Li, C. G. Lu, and X. Zhang, All-Optical Hall Effect by the Dynamic Toroidal Moment in a Cavity-Based Metamaterial, Phys. Rev. B 87, 245429 (2013).

[46] C. F. Bohren and D. R. Huffman, Absorption and Scattering of Light by Small Particles (Wiley-Interscience, New York, 1983).

[47] E. A. Semouchkina, G. B. Semouchkin, M. Lanagan, and C. A. Randall, FDTD Study of Resonance Processes in Metamaterials, IEEE Trans. Microwave Theory Tech. 53, 1477 (2005).

[48] M. S. Wheeler, J. S. Aitchison, and M. Mojahedi, ThreeDimensional Array of Dielectric Spheres with an Isotropic Negative Permeability at Infrared Frequencies, Phys. Rev. B 72, 193103 (2005).

[49] J. A. Schuller, R. Zia, T. Taubner, and M. L. Brongersma, Dielectric Metamaterials Based on Electric and Magnetic Resonances of Silicon Carbide Particles, Phys. Rev. Lett. 99, 107401 (2007).

[50] T. Lepetit, E. Akmansoy, and J. P. Ganne, Experimental Measurement of Negative Index in an All-Dielectric Metamaterial, Appl. Phys. Lett. 95, 121101 (2009).

[51] V. V. Khardikov, E. O. Larko, and S. L. Prosvirnin, A Giant Red Shift and Enhancement of the Light Confinement in a Planar Array of Dielectric Bars, J. Opt. 14, 035103 (2012).

[52] J. C. Ginn, I. Brener, D. W. Peters, J. R. Wendt, J. O. Stevens, P. F. Hines, L. I. Basilio, L. K. Warne, J. F. Ihlefeld, P. G. Clem, and M. B. Sinclair, Realizing Optical
Magnetism from Dielectric Metamaterials, Phys. Rev. Lett. 108, 097402 (2012).

[53] S. O'Brien and J. B. Pendry, Photonic Band-Gap Effects and Magnetic Activity in Dielectric Composites, J. Phys. Condens. Matter 14, 4035 (2002).

[54] L. Peng, L. X. Ran, H. S. Chen, H. F. Zhang, J. A. Kong, and T. M. Grzegorczyk, Experimental Observation of LeftHanded Behavior in an Array of Standard Dielectric Resonators, Phys. Rev. Lett. 98, 157403 (2007).

[55] J. B. Pendry, A. J. Holden, D. J. Robbins, and W. J. Stewart, Magnetism from Conductors and Enhanced Nonlinear Phenomena, IEEE Trans. Microwave Theory Tech. 47, 2075 (1999).

[56] V. Yannopapas and E. Paspalakis, Backward-Propagating Slow Light in Mie Resonance-Based Metamaterials, J. Opt. 12, 104017 (2010).

[57] T. Feurer, N. S. Stoyanov, D. W. Ward, J. C. Vaughan, E. R. Statz, and K.A. Nelson, in Annual Review of Materials Research (Annual Reviews, Palo Alto, 2007), p. 317-350.

[58] K. C. Huang, M. L. Povinelli, and J. D. Joannopoulos, Negative Effective Permeability in Polaritonic Photonic Crystals, Appl. Phys. Lett. 85, 543 (2004).

[59] A. S. Barker, Jr., A. A. Ballman, and J. A. Ditzenberger, Infrared Study of the Lattice Vibrations in $\mathrm{LiTaO}_{3}$, Phys. Rev. B 2, 4233 (1970).

[60] V. Savinov, V. A. Fedotov, and N. I. Zheludev, Toroidal Dipolar Excitation and Macroscopic Electromagnetic Properties of Metamaterials, Phys. Rev. B 89, 205112 (2014).

[61] J. D. Jackson, Classical Electrodynamics (Wiley, New York, 1962).

[62] H. C. Van der Hulst, Light Scattering by Small Particles (Wiley, New York, 1957). 